\title{
An unusual presentation of Crohn's disease
}

\author{
JA Keir, R McGregor, CJ Richards, R Windle \\ Departments of General Surgery and Pathology, Glenfield Hospital, Leicester, UK
}

\begin{abstract}
The first case of an inflamed, discharging urachal remnant associated with granulomatous appendicitis in which the patient was subsequently found to have Crohn's disease is described.
\end{abstract}

Key words: Urachal remnant - Granulomatous appendicitis - Crohn's disease

$\mathrm{T}^{\mathrm{s}}$ he urachus is a normal embryonic remnant of the allantoic duct that persists as a narrow cord between the bladder and the umbilicus. ${ }^{1}$ We present the first case of an inflamed, discharging urachal remnant associated with granulomatous appendicitis in which the patient was subsequently found to have Crohn's disease.

\section{Case report}

A 16-year-old girl presented with a 1 month history of a yellow fluid discharge from an ovoid $0.5 \mathrm{~cm}$ white granulomatous lesion in the umbilicus. No other symptoms were present at the time of presentation. Investigations revealed a raised $\mathrm{C}$-reactive protein at 31 and a microcytic anaemia (haemoglobin, $10.2 \mathrm{~g} / \mathrm{dl}$ and mean cell volume of 74). MRI demonstrated a patent urachus.

The patient underwent elective excision of the inflammatory mass, which turned out to be part of the urachus, the tract of which was freed and dissected down to the level of the bladder. A loop of terminal ileum together with an inflamed appendix was found to be adherent to the dilated tract and to the surface of the bladder. Consequently, an appendicectomy was also performed. The discharge settled postoperatively.

Histology of the appendix showed marked mural thickening with transmural lymphoid aggregates, focally active mucosal inflammation and occasional non- necrotising granulomata. At that time, a differential diagnosis of Yersinia spp. infection, idiopathic granulomatous appendicitis and Crohn's disease was given although the former was not considered likely. Yersinia serology was unfortunately not carried out at this time. The urachus consisted of a muscular tubal structure lined by granulation tissue with occasional abscesses and foreign body type giant cells but no granulomata (Fig. 1).

A year following discharge, the patient developed a wound abscess at the site of operation which was incised

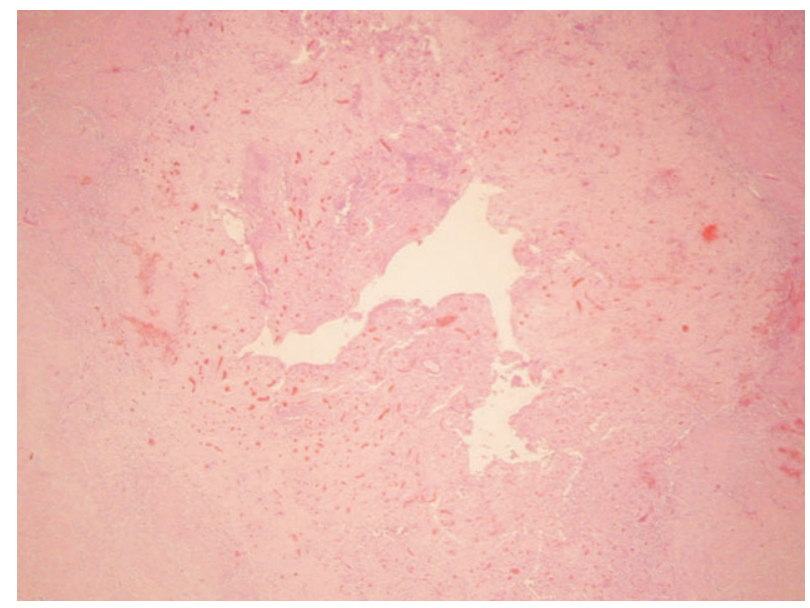

Figure I Histology of the urachus. 


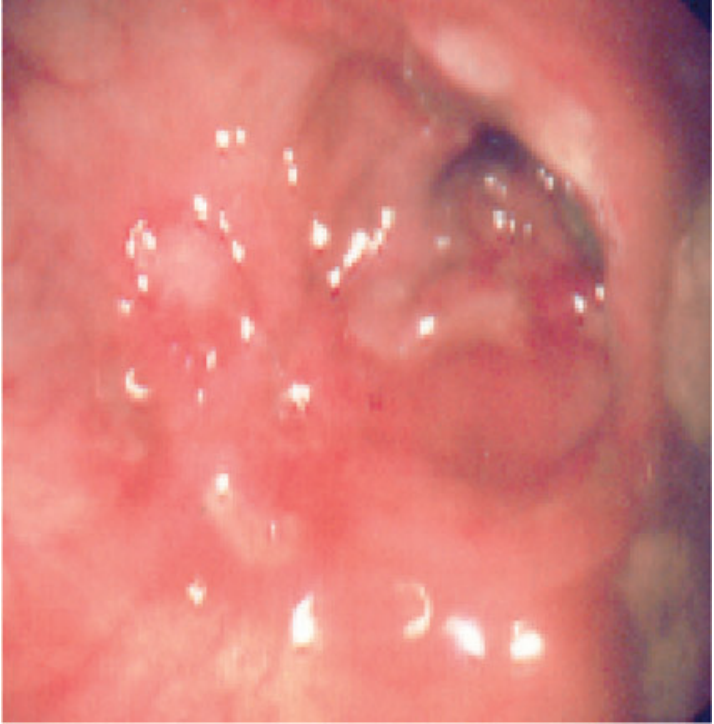

Figure 2 Caecal mucosa showing moderate patchy erythema, multiple large ulcers and a 'cobblestone' appearance at colonoscopy.

and drained. Afterwards, the wound remained unhealed and from time to time food was seen to appear within it, raising the question of a fistula. A sinogram was arranged which showed a communication with small bowel and a segment of small bowel containing a stricture. Further investigation of the stricture by $\mathrm{CT}$ found thickening of the terminal ileum. At colonoscopy, the caecal mucosa showed moderate patchy erythema, multiple large ulcers and a 'cobblestone' appearance (Fig. 2). Biopsies showed patchy active chronic inflammation consistent with Crohn's disease.
The patient was commenced on Azathioprine and has intermittently required antibiotics for recurrent infections at the site of the enterocutaneous fistula. Although the patient remained reasonably well, the fistula still discharged intermittently. A right hemicolectomy was, therefore, performed 2 years after initial presentation. There was a stricture of the terminal ileum and part of the caecum over a length of $8.5 \mathrm{~cm}$. The mucosa had a 'cobblestone' appearance with focal ulceration. Histology showed classical features of Crohn's disease. The resection margins showed no active disease. The patient was discharged home at a week and she was well with a healed wound when seen at 6 weeks.

\section{Discussion}

Urachal anomalies are rare with only 8 cases being identified among 40,000 admissions to a surgical unit in one study. ${ }^{2}$ Therefore, this serves as an unusual and interesting case. Though it is possible the infected urachal remnant and granulomatous appendicitis were coincidental findings, it is likely that the urachus had become infected secondary to intra-abdominal sepsis. To our knowledge, we present the first case of an inflamed urachal sinus and granulomatous appendicitis with the subsequent development of Crohn's disease.

\section{References}

1. Bauer SB, Retik AB. Urachal anomalies and related disorders. Urol Clin North Am 1978; 5: 195-211.

2. Blichert-Toft M, Koch F, Nielson OV. Anatomic variants of the urachus related to clinical appearance and surgical treatment of urachal lesions. Surg Gynecol Obstet 1973; 137: 51-4. 